\title{
What are the most important prognostic factors in patients with residual rectal cancer after preoperative chemoradiotherapy?
}

\author{
Sol-Min Kim ${ }^{1, *}$, Ghilsuk Yoon ${ }^{1,2, *}$, An Na Seo ${ }^{1,2}$ \\ ${ }^{1}$ Department of Pathology, School of Medicine, Kyungpook National University, Daegu, Korea \\ ${ }^{2}$ Department of Pathology, Kyungpook National University Chilgok Hospital, Daegu, Korea
}

Received: February 13, 2019

Revised: March 15, 2019

Accepted: March 18, 2019

Corresponding author:

An Na Seo

Department of Pathology, School of Medicine, Kyungpook National University, Kyungpook National University Chilgok Hospital, 807 Hoguk-ro, Buk-gu, Daegu 41404, Korea

Tel: $+82-53-200-3403$

Fax: +82-53-200-3399

E-mail:san_0729@naver.com

*These authors contributed to this work as co-first authors
Background: We aimed to establish robust histoprognostic predictors on residual rectal cancer after preoperative chemoradiotherapy (CRT).

Methods: Analyzing known histoprognostic factors in 146 patients with residual disease allows associations with patient outcome to be evaluated.

Results: The median follow-up time was 77.8 months, during which 59 patients (40.4\%) experienced recurrence and $41(28.1 \%)$ died of rectal cancer. On univariate analysis, residual tumor size, ypT category, ypN category, ypTNM stage, downstage, tumor regression grade, lymphatic invasion, perineural invasion, venous invasion, and circumferential resection margin (CRM) were significantly associated with recurrence free survival (RFS) or/and cancer-specific survival (CSS) (all $p<0.005$ ). On multivariate analysis, higher ypTNM stage and CRM positivity were identified as independent prognostic factors for RFS (ypTNM stage, $p=0.024$; CRM positivity, $p<0.001$ ) and CSS ( $p=0.022, p=0.017$, respectively). Furthermore, CRM positivity was an independent predictor of reduced RFS and CSS, irrespective of subgrouping according to downstage (non-downstage, $p<0.001$ and $p<0.001$; downstage, $p=0.002$ and $p=0.002$ ) or lymph node metastasis (non-metastasis, $p<0.001$ and $p=0.001$; metastasis, $p<0.001$ and $p<0.001$ ).

Conclusion: CRM status may be as powerful as ypTNM stage as a prognostic indicator for patient outcome in patients with residual rectal cancer after preoperative CRT.

Keywords: Circumferential resection margin; Preoperative chemoradiotherapy; Prognosis; Rectal cancer; Stage; Tumor regression grade

\section{Introduction}

Preoperative chemoradiotherapy (CRT) followed by principle of total mesorectal excision (TME) is now become the standard treatment for clinically staged, locally advanced mid- or low-rectal cancer, i.e. tumor with extension of cT3-T4 and/or the presence of suspected regional metastatic lymph nodes [1]. Preoperative CRT may facilitate complete radical resection negative surgical resection margin and sphincter preservation with by downsize and/or downstage of primary tumor [2], and allow an evaluation of chemo- or radio-sensitivity of tumor against the given therapeutic regimen [3]. Furthermore, the degree of response to preoperative CRT is regarded a prognostic indicator of patient outcome, and the patients achieving pathological complete response ( $\mathrm{pCR}$ ) after preoperative CRT have been showed excellent disease-free survival (DFS) [3]. However, pCR rate 
has been reported in $8-27 \%$ of patients receiving preoperative CRT [4-6], and the majority of patients were not achieved pCR. Therefore, it is great importance to identified prognostic factors of patients with residual rectal cancer after preoperative CRT.

Pathological prognostic factors, such as ypTNM stage, perineural invasion, lymphatic invasion, venous invasion, circumferential resection margin (CRM), and tumor regression grade (TRG), are easily identified but are still important for patient outcome and essential to determining further clinical management of patients [1,2,7-9]. Several studies investigated the association between pathological factors and patient outcome in rectal cancer patients treated with preoperative CRT, however, data from the previous studies on the prognostic value of pathological factor are inconsistent [1,2,10-12]. Quah et al. [2] demonstrated that "pathologic stage (ypTN stage)" was the most precise prognostic predictor. On the other hand, Gosens et al. [11] reported that CRM positivity and pathologic lymph node status were most significant factors and their combination might give stronger evidence for patient outcome than classic ypTNM stage. In addition, Huebner et al. [12] showed that TRG and pathologic lymph node status were relevant prognostic predictors. However, data from other studies on the prognostic value of TRG in rectal cancer patients received preoperative CRT are inconsistent $[2,9,12-14]$. Several factors could be responsible for these discrepancies, including inter-observer variation and different methods of quantifying for tumor regression. Unfortunately, most studies evaluated the prognostic significance of pathological factors in rectal cancer patients treated with preoperative CRT without consideration of $\mathrm{pCR}$. Because patients achieved $\mathrm{pCR}$ have all favorable histoprognostic status, including absence of lymphatic invasion, perineural invasion, and venous invasion, negative CRM, ypTONO (by seventh Union for International Cancer Control [UICC]/American Joint Committee on Cancer [AJCC $][15]$ ), prognostic value of analyzing proven pathological factors could be different in patients with residual disease after preoperative CRT. Therefore, robust pathological prognostic factors of residual disease will also need to be determined.

In this study, we investigated known histoprognostic factors in correlation with patient survival to identify robust prognostic predictors in patients with residual disease after preoperative CRT.

\section{Materials and methods}

\section{Patient population}

After the approval of the Institutional Review Boards of Kyungpook National University Medical Center, medical records of 146 patients with rectal cancer who underwent preoperative
CRT followed by TME at Kyungpook National University Medical Center between January 2006 and December 2011 were first corrected (IRB No: 2014-04-215). Patients with clinical evidence of distant metastases at the time of diagnosis and patients who were reported as $\mathrm{pCR}$ were excluded. All patients had a biopsy-proven diagnosis of primary rectal adenocarcinoma and were identified locally advanced tumors ( $\mathrm{cT} 3-4$ or $\mathrm{cN}+$ ). Hematoxylin and eosin (H\&E)-stained slides and formalinfixed, paraffin-embedded (FFPE) tumor samples from surgically resected specimens for each case were retrospectively retrieved.

\section{Preoperative chemoradiotherapy and surgery}

All patients received preoperative long-course radiotherapy (45 or 50 Gy over 5 weeks) plus 5-fluorouracil-based concomitant chemotherapy (intravenous bolus or continuous infusion) during radiotherapy. All patients were performed radical resection, according to the principle of TME, between 6 and 8 weeks after the completion of preoperative CRT. The detailed process of preoperative CRT followed by surgical protocol has been previously published $[16,17]$.

\section{Clinical assessment}

After surgical resection, patients were followed 3-month intervals for the first 2 postoperative years and 6-month intervals thereafter. The clinical workup included digital rectal examination, serum carcinoembryonic antigen (CEA), colonoscopy, computed tomography $(\mathrm{CT})$ of the abdomen and pelvis or pelvic magnetic resonance imaging, and whole-body positron emission tomography/CT [16].

The recurrence free survival (RFS) was calculated from the time of surgery until the time of any recurrence of rectal cancer, and the cancer-specific survival (CSS) was calculated from the date of surgery to the date of death caused by rectal cancer or the date of last contact, as applicable.

\section{Pathological assessment and tumor regression grade}

For each surgically resected specimen, tumor size and distance of tumor from distal resection margin were measured at the time of gross examination. The non-peritonealized surface of the surgical specimen was painted with green ink to facilitate recognition of CRM. The specimen was opened along the anterior aspect and fixed in 10\% neutral-buffered formalin overnight [18]. The entire tumor site was serially sectioned and embedded in paraffin, and 4- $\mu \mathrm{m}$ sections were cut and stained with H\&E [1]. All H\&Estained slides were retrospectively reviewed by two pathologists (A.N.S and G.S.Y) blinded to the patients' clinical data and reevaluated pathological characteristics, as follows: tumor size; 
presence of lymphatic invasion, perineural invasion, and venous invasion; ypT stage, ypN stage, and ypTNM stage; TRG; and CRM. The tumors stage was determined according to the UICC/ AJCC staging manual for colorectal cancer (7th edition) [15]. Tumor regression of the primary tumor against preoperative CRT was semi-quantitatively assessed using 5-point TRG system as initially described by Rödel et al. [3], as follows: grade 0 , no regression; grade 1 , minor regression ( $<25 \%$ of tumor mass); grade 2 , moderate regression ( $\geq 25 \%$ to $50 \%$ of tumor mass); grade 3 , good regression ( $>50 \%$ of tumor mass); and grade 4 (complete remission or pCR). A tumor with acellular mucin pools in whole tissue was considered as $\mathrm{PCR}$ and was excluded in the present study. In patients with non-pCR, inter-observer reproducibility for TRG was analyzed. Final consensus in cases showing discrepancies for TRG was reached in common session using the multi-head microscope. The CRM was measured as the shorter distance from the outermost part of the tumor cells to the inked resection margin. To evaluate the exact length, the CRM was measured by a ruler or a microscope graticule, and CRM positivity was defined as $\leq 1 \mathrm{~mm}$ [19]. If cases with CRMs of 1 to $2 \mathrm{~mm}$ were found, additional multiple-level serial and deep sections were examined in these cases to detect CRM positivity by hidden malignancy.

\section{KRAS and BRAF mutation}

Deoxyribonucleic acid (DNA) was extracted from FFPE representative tumor tissues using Maxwell 16FFPE Tissue LEV DNA Purification kit (Promega, Seoul, Korea) and identified for the presence of mutations in KRAS exon 2 (codon 12 and 13) and in BRAF V600E using the PNAClamp ${ }^{\mathrm{TM}} \mathrm{KRAS}$ and PNAClamp ${ }^{\mathrm{TM}}$ BRAF mutation Detection kit (Panagene, Daejeon, Korea) in PNA-mediated real-time polymerase chain reaction (PCR) as described previously [20]. The efficiency of PCR clamping was calculated by measuring the threshold cycle $(\mathrm{Ct})$ value. Ct values for control and mutation assays were automatically evaluated from SYBR Green amplification plots and delta-Ct values were calculated (control $\mathrm{Ct}-$ sample $\mathrm{Ct}$ ). A higher delta-Ct value shows that the mutant was efficiently amplified. The cut-off delta-Ct was defined as 2 for KRAS and BRAF mutation.

\section{Statistical analyses}

All statistical analyses were performed using IBM SPSS version 19.0 (IBM Co., Armonk, NY, USA). To evaluate the interobserver agreement for TRG, Cohen's Kappa $(\kappa)$ was used. Significant differences for survival between the two groups were compared using the log-rank test, and survival curves were plotted using the Kapan-Meier method. Multivariate analyses were performed using the Cox proportional hazard regression model to verify the independent prognostic impact for each factor. For multivariate analyses, covariates that were proven to be significant in the univariate analysis were controlled, and any possibility of multicollinearity was excluded from the final model. The hazard ratio (HR) and $95 \%$ confidence interval (CI) were assessed for each factor. All tests were two-sided, and statistical significance was considered as $p<0.05$.

\section{Results}

\section{Patients' clinicopathologic characteristics}

A total of 146 patients with residual disease in the surgically resected rectal cancer after preoperative CRT were analyzed. The baseline characteristics of patients are provided in Table 1 . The patient group included $102(69.9 \%)$ male and 44 (30.1\%) female with median age of 60.0 years (range, 29-85 years). The majority of patients were identified tumor distance from anal verge of $<5$ $\mathrm{cm}(76.7 \%)$ and pretreatment serum CEA level of $\leq 5 \mathrm{ng} / \mathrm{mL}$ (66.9\%). At the time of initial diagnosis, 17 (11.6\%) patients and $129(88.4 \%)$ had evidence of cTNM stage II and III, respectively.

On gross examination, residual tumor size was ranged from 2.3 to $10.0 \mathrm{~cm}$ (median range, $6.0 \mathrm{~cm}$ ). At the time of surgical resection, 31 tumors (21.2\%) were ypTMN stage I, 68 tumors (46.6\%) were stage II, and 47 tumors (32.2\%) were stage III. When comparing with cTNM stage, 92 (63.0\%) patients were experienced downstage after preoperative CRT. For the degree of response to preoperative CRT, TRG 0 was found in 4 of 146 (2.7\%) patients, TRG 1 in 21 (14.4\%), TRG 2 in 55 (37.7\%), and TRG 3 in 66 (45.2\%). All cases were independently re-evaluated TRG by two pathologists, and inter-observer agreement was analyzed. When comparing an each TRG, the inter-observer agreement showed a moderate agreement ( $\kappa=0.508 ; p<0.001$ ), whereas, when comparing dichotomous groups (TRG $0 \& 1$ vs. TRG $2 \& 3$ ), kappa value reached 0.534 $(p<0.001)$. In addition, CRM positivity was observed in 30 of 146 (20.5\%) patients. CRM positivity was more commonly identified in adverse ypT category tumors $(p=0.001)$, adverse ypTNM stage tumors ( $p=0.002)$, tumors with lower TRG, and tumors with perineural invasion $(p=0.058)$. Of the 146 patients, mutation testing was available for 105 patients (72.4\%), 29 (27.6\%) and 4 (3.8\%) were identified to have KRAS mutant and BRAF mutant primary tumors, respectively.

\section{Prognostic factors and univariate analyses}

At the time last analyses (December 2017), the median follow-up time was 77.8 months (range, 3.1-141.7 months), during which 59 patients (40.4\%) experienced recurrence and 41 (28.1\%) died 
Table 1. Characteristics of the patients with residual rectal cancer after preoperative CRT $(n=146)$

\begin{tabular}{|c|c|c|}
\hline Characteristic & $n$ & $\%$ \\
\hline \multicolumn{3}{|c|}{ Age at diagnosis (yr) } \\
\hline Median & \multicolumn{2}{|c|}{60} \\
\hline Range & \multicolumn{2}{|c|}{$29-85$} \\
\hline \multicolumn{3}{|l|}{ Sex } \\
\hline Male & 102 & 69.9 \\
\hline Female & 44 & 30.1 \\
\hline \multicolumn{3}{|c|}{ Tumor distance from anal verge $(\mathrm{cm})$} \\
\hline$<5$ & 112 & 76.7 \\
\hline$\geq 5$ & 34 & 23.3 \\
\hline \multicolumn{3}{|c|}{ Residual tumor size $(\mathrm{cm})$} \\
\hline Median & \multicolumn{2}{|c|}{6.0} \\
\hline Range & \multicolumn{2}{|c|}{$2.3-10.0$} \\
\hline \multicolumn{3}{|c|}{ Clinical T category } \\
\hline cT2 & 8 & 5.5 \\
\hline cT3 & 121 & 82.9 \\
\hline cT4 & 17 & 11.6 \\
\hline \multicolumn{3}{|c|}{ Clinical $\mathrm{N}$ category } \\
\hline cNO & 17 & 11.6 \\
\hline cN1-3 & 129 & 88.4 \\
\hline \multicolumn{3}{|c|}{ Preoperative CEA (ng/mL) } \\
\hline$\leq 5$ & 91 & 66.9 \\
\hline$>5$ & 45 & 33.1 \\
\hline \multicolumn{3}{|c|}{ Tumor regression grade } \\
\hline 0 & 4 & 2.7 \\
\hline 1 & 21 & 14.4 \\
\hline 2 & 55 & 37.7 \\
\hline 3 & 66 & 45.2 \\
\hline \multicolumn{3}{|c|}{ Pathologic TNM stage } \\
\hline I & 31 & 21.2 \\
\hline$\|$ & 68 & 46.6 \\
\hline III & 47 & 32.2 \\
\hline \multicolumn{3}{|c|}{ Down-staged after CRT } \\
\hline No & 54 & 37 \\
\hline Yes & 92 & 63 \\
\hline \multicolumn{3}{|c|}{ KRAS mutation ${ }^{\text {a) }}$} \\
\hline Negative & 76 & 72.4 \\
\hline Positive & 29 & 27.6 \\
\hline \multicolumn{3}{|c|}{$B R A F$ mutation $^{\text {a) }}$} \\
\hline Negative & 101 & 96.2 \\
\hline Positive & 4 & 3.8 \\
\hline
\end{tabular}

CRT, chemoradiotherapy; CEA, carcinoembryonic antigen; TNM, tumor, node, metastasis.

${ }^{\text {a) }}$ Missing value is included.

of rectal cancer. During follow-up period, two patients treated with cetuximab (epidermal growth factor receptor monoclonal antibody) for their metachronous metastases. The actuarial 5-year RFS and 5-year CSS were $62.0 \%$ and $76.0 \%$, respectively.
In univariate analysis, the following 10 factors were identified as being significantly associated with shorter RFS or/and CSS: larger residual tumor size; higher ypT category, ypN category, and ypTNM stage; non-downstage after preoperative CRT; lower TRG; presence of lymphatic invasion, perineural invasion, and venous invasion; and CRM positivity (all $p<0.005$, Table 2; Fig. 1 ). Unfortunately, KRAS mutation-positivity and $B R A F$ mutationpositivity were not associated with RFS (KRAS, $p=0.107$; $B R A F$, $p=0.435$ ) and CSS (KRAS, $p=0.122 ; B R A F, p=0.186$ ). Then, we performed subgroup analyses according to downstage and lymph node metastasis (LNM) status after preoperative CRT, respectively. As shown Table 3, in patients subgroup showing nondownstage $(\mathrm{n}=54)$, more advanced ypT category, lower TRG, presence of lymphatic invasion, perineural invasion, and venous invasion, and CRM positivity (Figs. 2A, 2B) were related to worse RFS or CSS, and both. In patients subgroup showing downstage $(\mathrm{n}=92)$, larger residual tumor size, higher $y \mathrm{pT}$ category, and CRM positivity (Figs. 2C, 2D) were correlated with poorer RFS or/and CSS. On the other hand, in patients without LNM ( $n=99)$, larger residual tumor size, higher $\mathrm{ypT}$ category, and CRM positivity (Figs. 2E, 2F) were associated with shorter RFS or CSS, and both (Table 3). In patients with LNM ( $\mathrm{n}=47)$, more advance ypT category, lower TRG, presence of perineural invasion and venous invasion, and CRM positivity (Figs. 2G, 2H) correlated with worse RFS or/and CSS (Table 3).

\section{Multivariate analyses}

As shown Table 4, multivariate analyses revealed the following independent unfavorable prognostic factors for RFS: larger residual tumor size $(\mathrm{HR}=1.753 ; p=0.049)$, $\mathrm{CRM}$ positivity $(\mathrm{HR}=3.613 ; p<0.001)$, and presence of venous invasion $(\mathrm{HR}=20.425 ; p<0.001)$; and for CSS: higher ypTNM stage $(\mathrm{HR}=3.413 ; p=0.004)$, larger residual tumor size $(\mathrm{HR}=2.371$; $p=0.020)$ presence of perineural invasion $(\mathrm{HR}=2.634 ; p=0.009)$, and $\mathrm{CRM}$ positivity $(\mathrm{HR}=4.133 ; p<0.001)$.

Then, we also evaluated the independency of prognostic factors within aforementioned subgroups. In patients with nondownstage, for RFS, presence of venous invasion (HR=18.066; $p<0.001)$ and CRM positivity $(\mathrm{HR}=3.715 ; p=0.004)$; for CSS, presence of venous invasion $(\mathrm{HR}=4.072 ; p=0.023)$, perineural invasion $(\mathrm{HR}=3.067 ; p=0.023)$, and $\mathrm{CRM}$ positivity $(\mathrm{HR}=2.666 ; p=0.043)$ were independent negative prognostic factors, respectively. While, in patients with downstage, for RFS, CRM positivity ( $\mathrm{HR}=2.388$; $p=0.028)$; for CSS, larger residual tumor size $(\mathrm{HR}=3.599 ; p=0.028)$ and $\mathrm{CRM}$ positivity $(\mathrm{HR}=3.829 ; p=0.019)$ were independent adverse prognostic factors, respectively. Additionally, in patients without lymph node 
Table 2. Univariate analysis of prognostic factors in entire group with residual rectal cancer patients after preoperative CRT

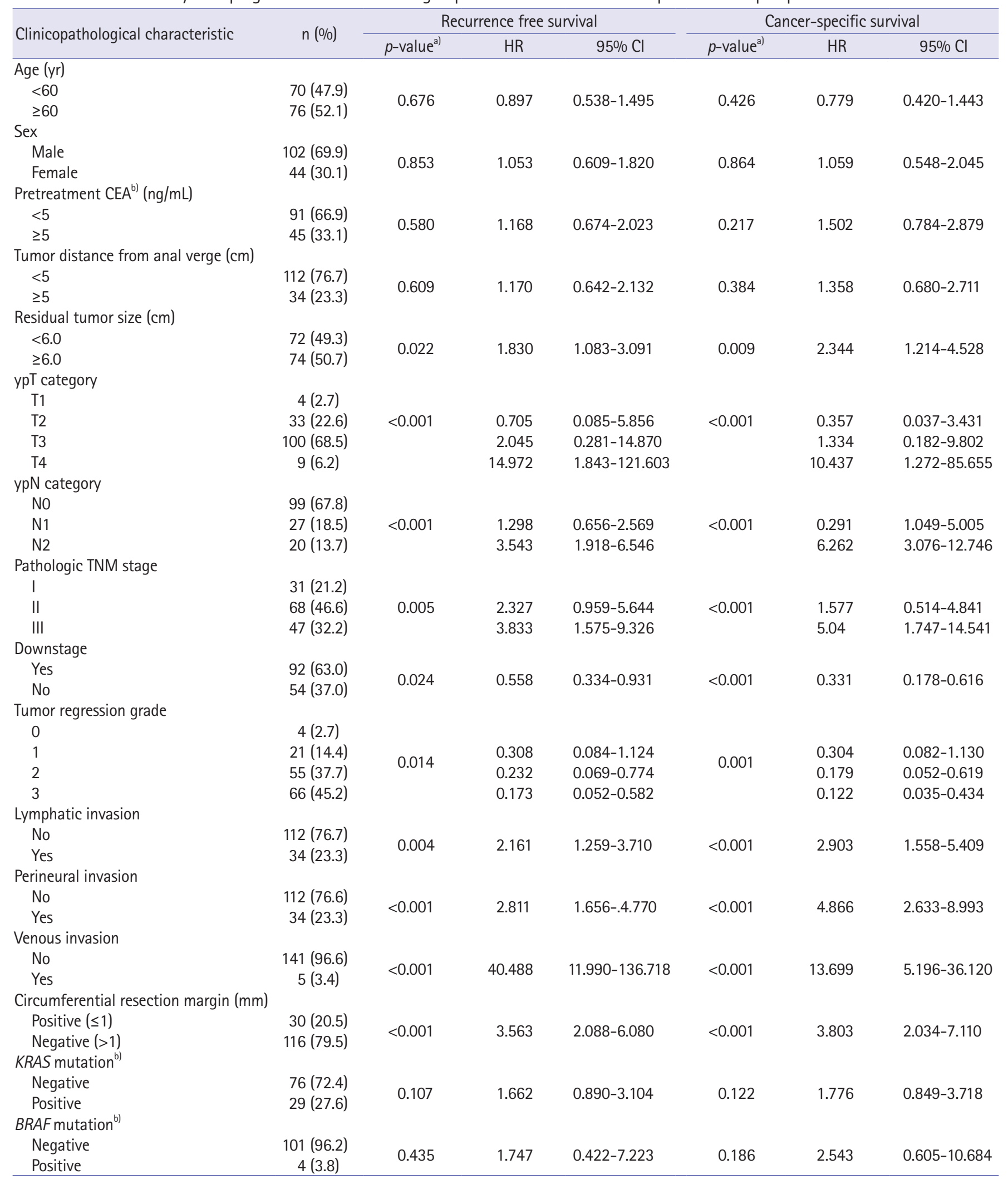

CEA, carcinoembryonic antigen; CRT, chemoradiotherapy; HR, hazard ratio; $\mathrm{Cl}$, confidence interval; TNM, tumor, node, metastasis.

${ }^{\text {a) }} p$-values were calculated using log-rank test. ${ }^{\text {b) }}$ Missing value is included. 


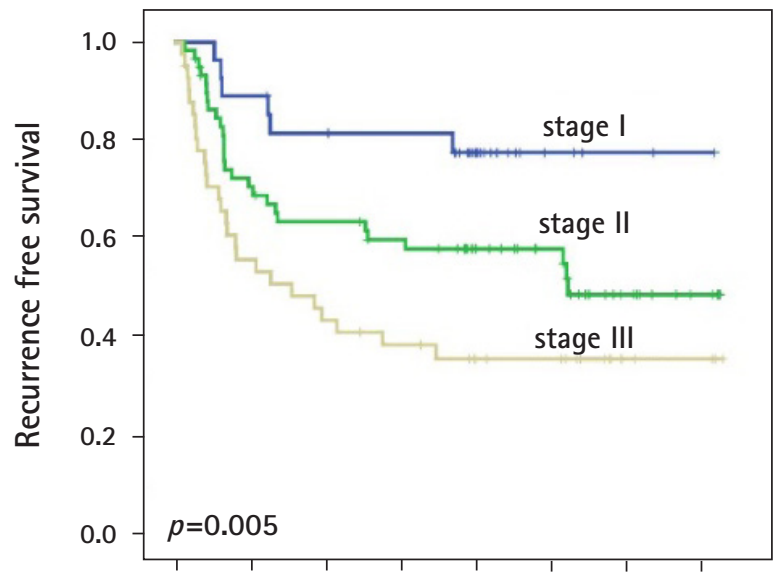

(A) $\begin{array}{llllllll}0.0 & 20.0 & 40.0 & 60.0 & 80.0 & 100.0 & 120.0 & 140.0\end{array}$ Follow-up time (mon)

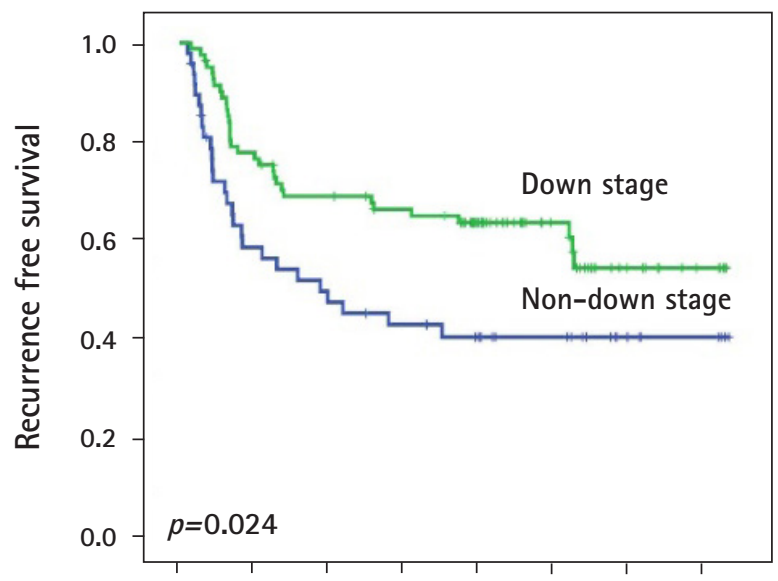

C $\quad \begin{array}{llllllll}0.0 & 20.0 & 40.0 & 60.0 & 80.0 & 100.0 & 120.0 & 140.0\end{array}$ Follow-up time (mon)

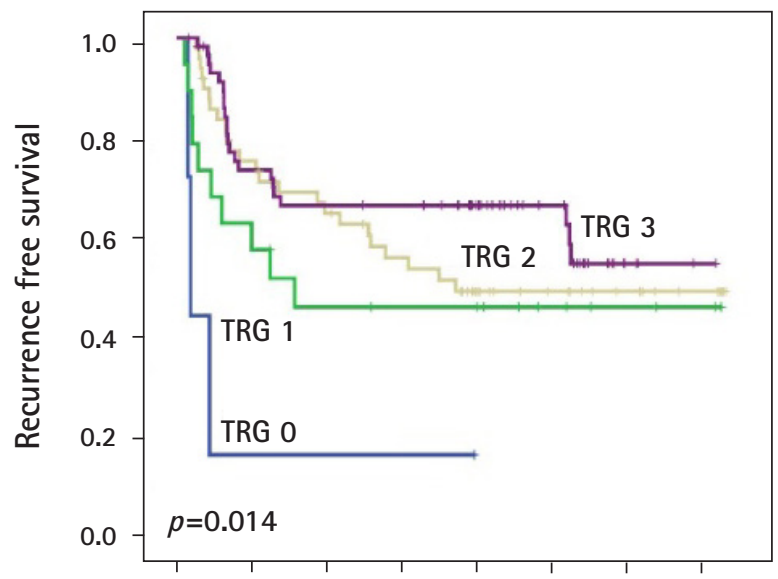

E

Follow-up time (mon)

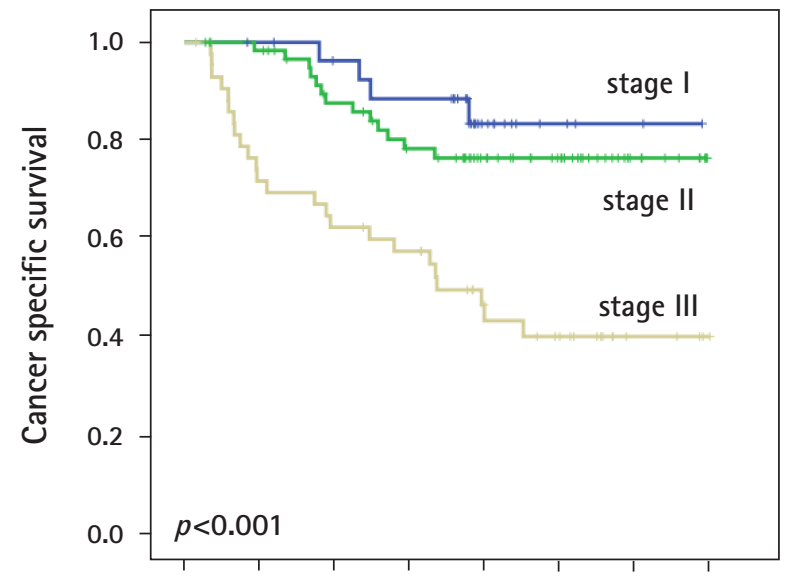

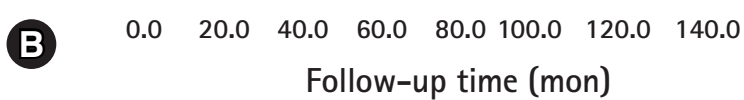

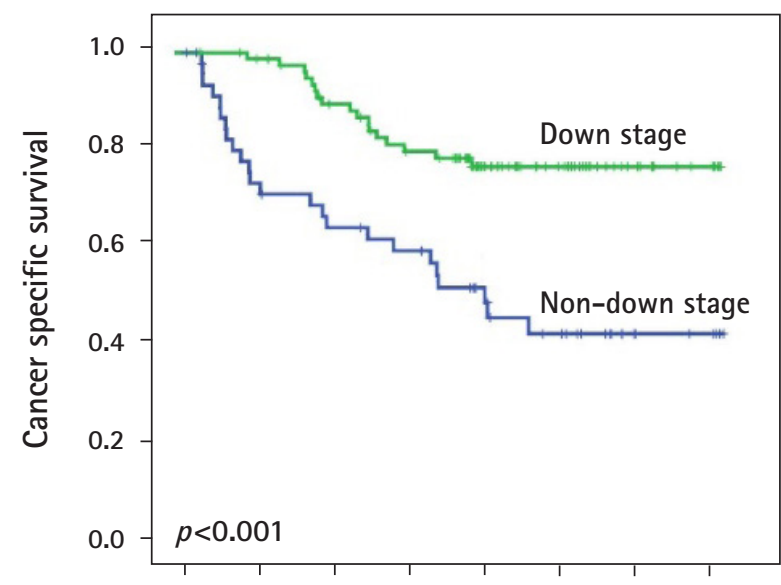

D $\quad \begin{array}{llllllll}0.0 & 20.0 & 40.0 & 60.0 & 80.0 & 100.0 & 120.0 & 140.0\end{array}$ Follow-up time (mon)

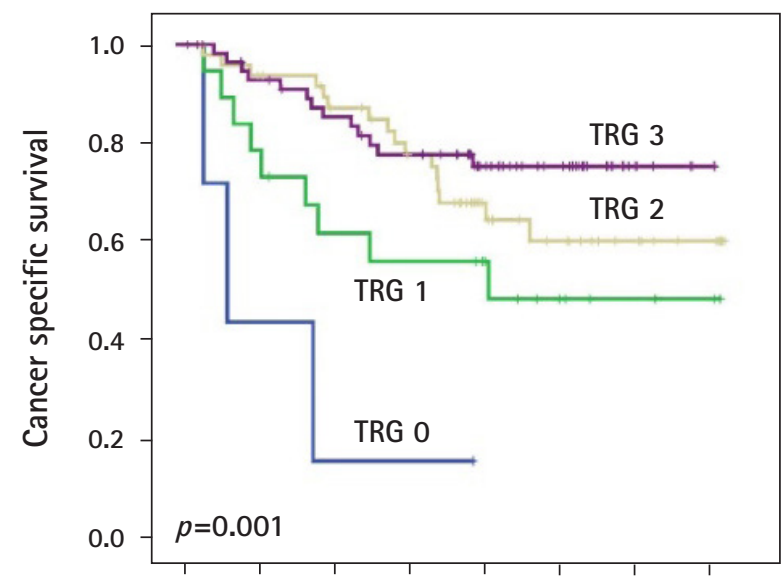

F $\quad \begin{array}{llllllll}0.0 & 20.0 & 40.0 & 60.0 & 80.0 & 100.0 & 120.0 & 140.0\end{array}$ Follow-up time (mon) 

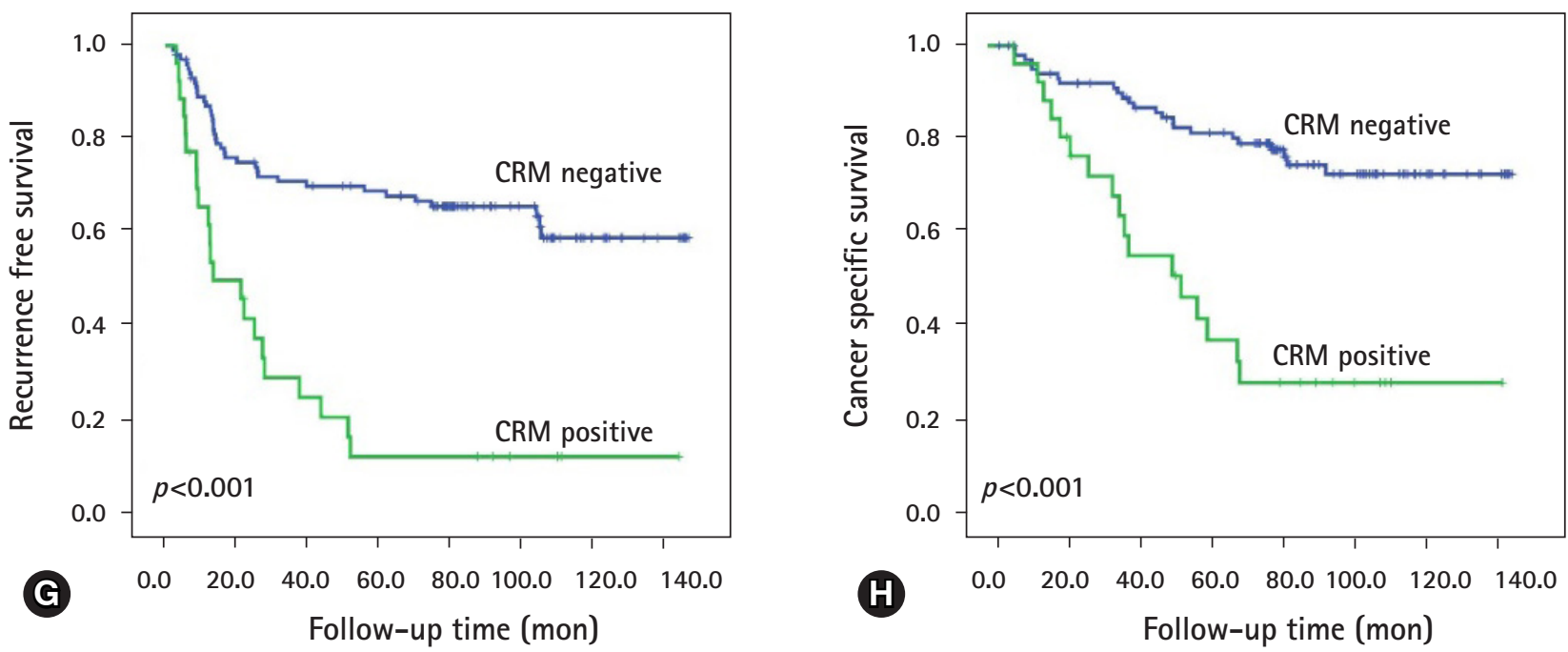

Fig. 1. Survival estimates of representative histopathologic factors in residual rectal cancer after preoperative chemoradiotherapy using the Kaplan-Meier method. ypTNM stage for (A) RFS and (B) CSS; down stage for (C) RFS and (D) CSS; tumor regression grade (E) RFS and (F) CSS; and CRM for (G) RFS and (H) CSS. RFS, recurrence free survival; CSS, cancer specific survival; CRM, circumferential resection margin; TRG, tumor regression grade.

metastasis, for RFS, CRM positivity ( $\mathrm{HR}=2.841 ; p=0.007)$; for CSS, larger residual tumor size $(\mathrm{HR}=3.589 ; p=0.028)$ and CRM $(\mathrm{HR}=4.257 ; p=0.011)$ were independent worse prognostic factors, respectively. Whereas, in patients with LNM, for RFS, CRM positivity ( $\mathrm{HR}=3.011 ; p=0.005)$; for CSS, presence of perineural invasion $(\mathrm{HR}=2.973 ; p=0.027)$ and $\mathrm{CRM}$ positivity $(\mathrm{HR}=3.053 ; p=0.018)$ were independent negative prognostic factors, respectively.

\section{Discussion}

Accurate pathological examination of surgical specimens resected from rectal cancer patients treated with preoperative CRT is crucial in determining prognostic factors, such as $\mathrm{pCR}$, that may affect patient outcome and subsequent therapeutic management. However, robust pathological factors of residual disease associated with patient outcome have yet to be fully elucidated. Therefore, analyzing known histoprognostic factors in patients with residual disease allows correlations with patient outcome to be evaluated. In our study, we investigated associations between histoprognostic factors and survival in patients with residual rectal cancer after surgical resection, who had received preoperative CRT. Our results revealed that residual tumor size, ypT category, ypN category, ypTNM stage, downstage, TRG, lymphatic invasion, perineural invasion, venous invasion, and CRM were significantly associated with RFS and/or CSS in patients with residual rectal cancer after preoperative CRT. Of these factors, CRM positivity was identified as an independent prognostic factor associated with poorer outcome with respect to RFS and CSS. Furthermore, CRM positivity was an independent predictor of reduced RFS and CSS, irrespective of subgrouping according to downstage or LNM. These findings are in strong accordance with the results of previous studies of rectal cancer patients treated with preoperative CRT, irrespective of pCR $[2,10,11,21]$. In these studies, Quah et al. [2] reported that "pathologic stage (ypTN stage)" was the most accurate predictor for DFS, while Gosens et al. [10] demonstrated that CRM positivity, lymph node status, and ypTNM stage were significantly associated with both local recurrence and overall survival on univariate analysis. In the latter study, CRM positivity was an independent predictor of local recurrence, but not of overall survival. In contrast, we determined that CRM positivity was an independent prognostic factor of both RFS and CSS, along with the ypTNM stage. Interestingly, a new staging system based on the combination of ypN stage and CRM status proposed by Gosens et al. was found to be a better prognostic tool than classic ypTNM staging in rectal cancer [11].

There is increasing evidence to suggest that CRM in rectal cancer is an important predictor of local recurrence, development of distant metastasis, and patient outcome [10,21-25]. While many studies have used the standard definition of $\leq 1 \mathrm{~mm}$ as a CRM positivity $[22,26]$, others have reported that tumor cells within $2 \mathrm{~mm}$ from the margin were associated with an unfavorable prognosis $[27,28]$. In this regard, by comparing cutoff values in rectal cancer patients who had received preoperative CRT, 

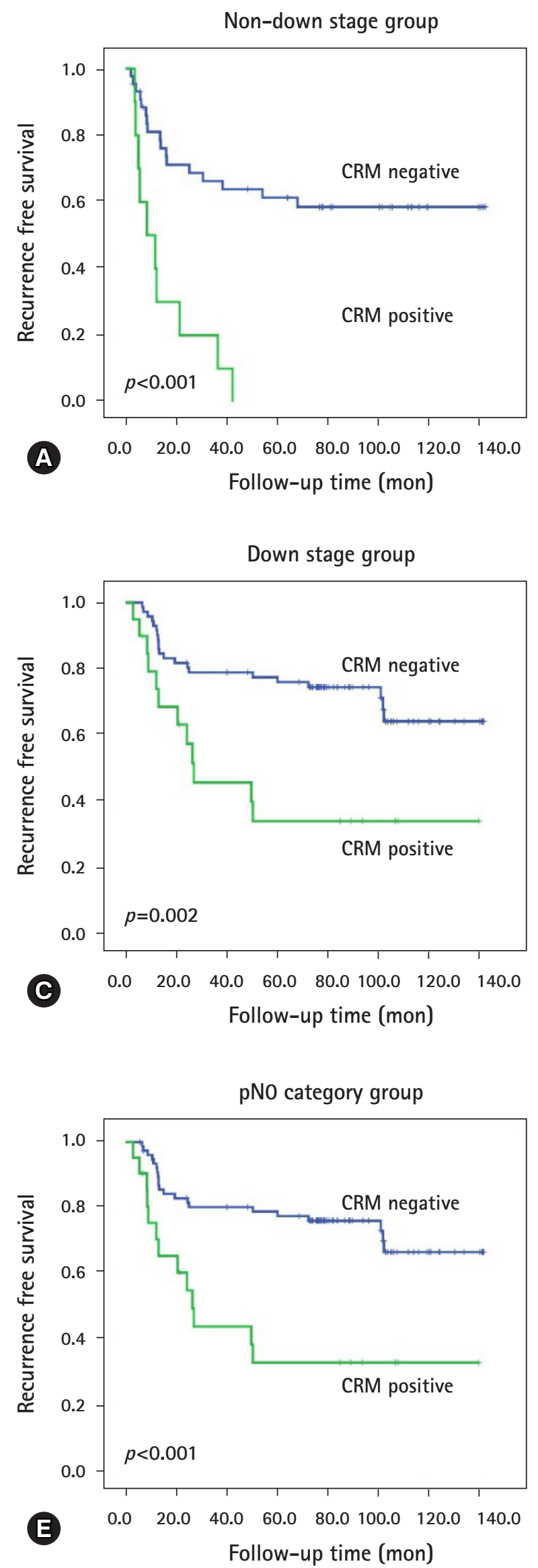
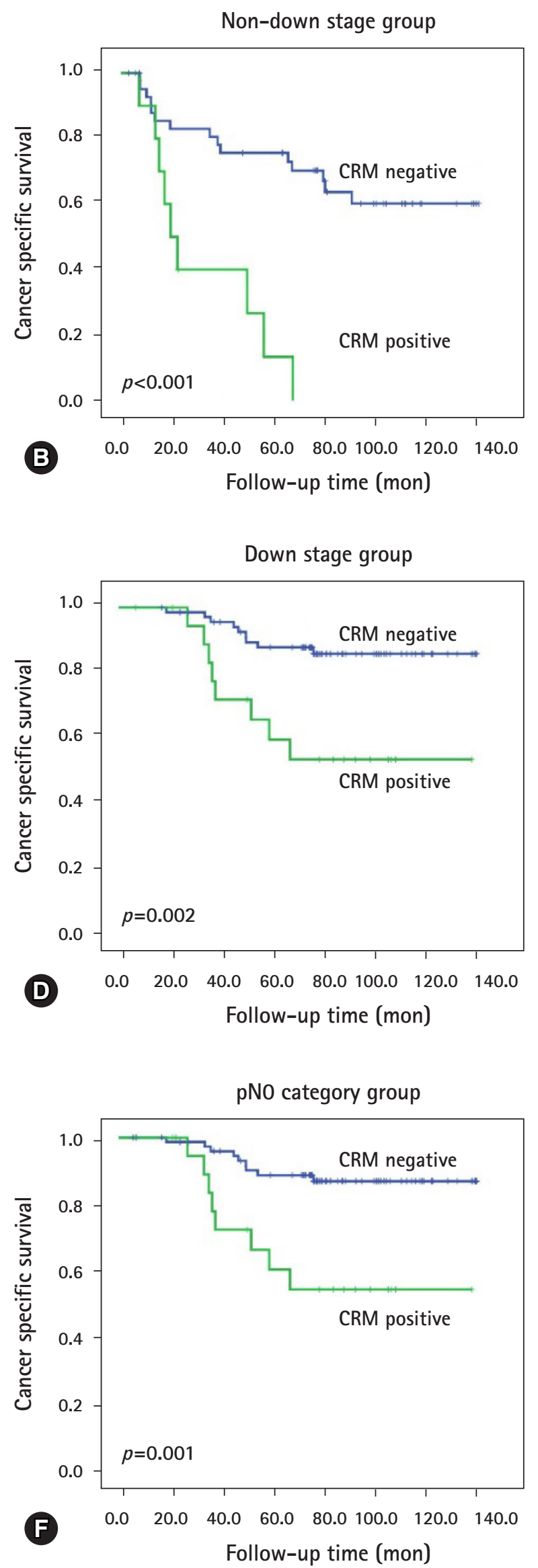

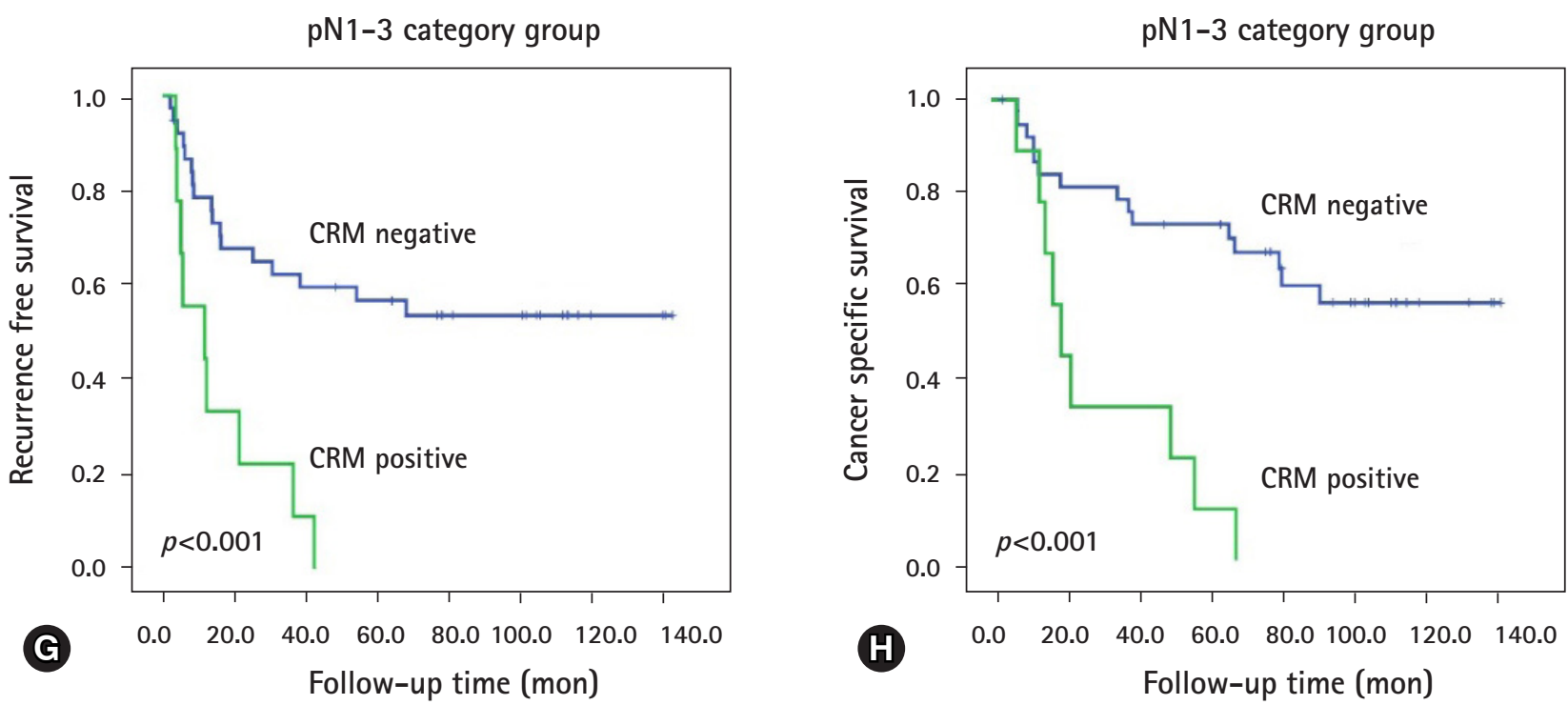

Fig. 2. Prognostic values of CRM on residual rectal cancer after preoperative chemoradiotherapy in subgroup analysis. Kaplan-Meier survival curves of (A) RFS and (B) CSS for patients without downstaging; and (C) RFS and (D) CSS for patients with downstaging. Kaplan-Meier survival curves of (E) RFS and (F) CSS for patients with pathologic No category; and (G) RFS and (H) CSS for patients with pathologic N1-3 category. CRM, circumferential resection margin; RFS, recurrence free survival; CSS, cancer specific survival;

Hwang et al. [19] demonstrated that $1 \mathrm{~mm}$ was an appropriate measurement for determining CRM positivity. Furthermore, Park et al. [18] demonstrated that a $1 \mathrm{~mm}$ cutoff value could be used to discern CRM positivity used in the prognosis of patient outcome, in rectal cancer patients with and without preoperative CRT. In our study, CRM positivity was defined as $\leq 1 \mathrm{~mm}$ and was observed in $20.5 \%$. Because our cohort was constructed solely from patients with residual disease, the percentage of CRM positivity was relatively high, although within the reported range of $4-21 \%[2,10,18,19,29]$. Our data suggest that CRM status may be as powerful as ypTNM staging as a prognostic indicator for patient outcome in patients with residual rectal cancer after preoperative CRT. Although CRM status has known prognostic value, we noted that CRM data were occasionally absent from pathology reports and they could not be assessed from paraffin blocks owing to a lack of embedding all clinically relevant areas. In addition, owing to the fibrotic response to CRT, tumor depth could not be accurately determined on gross examination of the surgical specimen of some patients. Furthermore, it has also been reported that vital tumor cells can be found scattered throughout the whole fibrotic area, resulting in CRM positivity [10]. Thus, we recommend that in the case of preoperative CRT rectal cancers, CRM assessment should be considered for all tissue surrounding the entire tumor CRM, and be documented within the pathology report. In addition, the pathologists should not hesitate to determine CRM positivity and the clinicians should not be afraid it to decide accurate treatment strategies.

Interestingly in our study, TRG is associated with patient outcome but is not an independent prognostic factor in patients with residual rectal cancer after CRT. In addition, we observed only moderate reproducibility of TRG scoring between two pathologists, which was complicated by evaluating TRG solely in patients with residual tumor, also observed in a previous study [10]. This discrepancy highlights that using TRG to predict patient outcome in residual rectal cancer presents with some difficulty in practice.

We acknowledge that the data presented: (1) is a retrospective study from a single institution with a relatively homogeneous population; and (2) may have too few numbers in each patient subgroup to perform robust statistical analyses. Therefore, subsequent large-scale studies are needed to validate our results. Despite these limitations, this study is a comprehensive evaluation of clinicopathological prognostic factors associated with residual rectal cancer after preoperative CRT in an East Asian population.

In conclusion, CRM positivity was independent prognostic indicator that predicted for poorer patient outcome in relation to both RFS and CSS irrespective of ypTNM stage, in patients with residual rectal cancer who received preoperative CRT. Additionally, in these patients, CRM positivity retained significance as an independent prognostic indicator of poor outcome for RFS and CSS, irrespective of downstage or LNM. Finally, these results have implications for the stratification of 
Table 3. Univariate analysis of prognostic factors in subgrouping patients according to downstage and LNM after preoperative CRT

\begin{tabular}{|c|c|c|c|c|c|c|c|c|c|c|c|c|}
\hline \multirow{2}{*}{$\begin{array}{l}\text { Clinicopathological } \\
\text { characteristic }\end{array}$} & \multicolumn{3}{|c|}{ Non-downstage group } & \multicolumn{3}{|c|}{ Downstage group } & \multicolumn{3}{|c|}{ non LNM group } & \multicolumn{3}{|c|}{ LNM group } \\
\hline & n $(\%)$ & \multicolumn{2}{|c|}{$p$-value ${ }^{a}$} & n (\%) & \multicolumn{2}{|c|}{$p$-value ${ }^{\text {a) }}$} & n $(\%)$ & \multicolumn{2}{|c|}{$p$-value ${ }^{a)}$} & n $(\%)$ & \multicolumn{2}{|c|}{$p$-value ${ }^{a)}$} \\
\hline \multicolumn{13}{|c|}{ Pretreatment CEA $(\mathrm{ng} / \mathrm{mL})^{\mathrm{b})}$} \\
\hline \multicolumn{13}{|c|}{$\begin{array}{l}\text { Tumor distance from anal } \\
\text { verge }(\mathrm{cm})\end{array}$} \\
\hline \multicolumn{13}{|c|}{ Residual tumor size (cm) } \\
\hline $\begin{array}{l}<6.0 \\
\geq 6.0\end{array}$ & $\begin{array}{l}23(42.6) \\
31(57.4)\end{array}$ & 0.451 & 0.354 & $\begin{array}{l}49(53.3) \\
43(46.7)\end{array}$ & 0.024 & 0.008 & $\begin{array}{l}52(52.5) \\
47(47.5)\end{array}$ & 0.018 & 0.009 & $\begin{array}{l}20(42.6) \\
27(57.4)\end{array}$ & 0.566 & 0.331 \\
\hline \multicolumn{13}{|l|}{ урT category } \\
\hline $\begin{array}{l}\text { T1 } \\
\text { T2 } \\
\text { T3 } \\
\text { T4 }\end{array}$ & $\begin{array}{c}0(0) \\
6(11.1) \\
41(75.9) \\
7(10.0)\end{array}$ & $<0.001$ & $<0.001$ & $\begin{array}{c}4(4.3) \\
27(29.3) \\
59(64.1) \\
2(2.2)\end{array}$ & 0.003 & 0.406 & $\begin{array}{c}4(4.0) \\
27(27.3) \\
66(66.7) \\
2(2.0)\end{array}$ & 0.004 & 0.414 & $\begin{array}{c}0(0) \\
6(12.8) \\
34(72.3) \\
7(14.9)\end{array}$ & $<0.001$ & $<0.001$ \\
\hline 0 & $2(3.7)$ & & & $2(2.2)$ & & & $2(2.0)$ & & & $2(4.3)$ & & \\
\hline $\begin{array}{l}1 \\
2 \\
3\end{array}$ & $\begin{array}{r}8(14.8) \\
22(40.7) \\
22(40.7)\end{array}$ & $<0.001$ & $<0.001$ & $\begin{array}{l}13(14.1) \\
33(35.9) \\
44(47.8)\end{array}$ & 0.845 & 0.397 & $\begin{array}{l}13(13.1) \\
26(36.4) \\
48(48.5)\end{array}$ & 0.846 & 0.348 & $\begin{array}{r}8(17.0) \\
19(40.4) \\
18(38.3)\end{array}$ & $<0.001$ & $<0.001$ \\
\hline \multicolumn{13}{|l|}{ Lymphatic invasion } \\
\hline $\begin{array}{l}\text { No } \\
\text { YES }\end{array}$ & $\begin{array}{l}25(46.3) \\
29(53.7)\end{array}$ & 0.041 & 0.035 & $\begin{array}{c}87(94.6) \\
5(5.4)\end{array}$ & 0.924 & 0.287 & $\begin{array}{c}94(94.9) \\
5(5.1)\end{array}$ & 0.964 & 0.301 & $\begin{array}{l}18(38.3) \\
29(61.7)\end{array}$ & 0.12 & 0.144 \\
\hline \multicolumn{13}{|l|}{ Perineural invasion } \\
\hline $\begin{array}{l}\text { No } \\
\text { YES }\end{array}$ & $\begin{array}{l}32(59.3) \\
22(40.7)\end{array}$ & $<0.001$ & $<0.001$ & $\begin{array}{l}80(87.0) \\
12(13.0)\end{array}$ & 0.286 & 0.105 & $\begin{array}{l}86(86.9) \\
13(13.1)\end{array}$ & 0.409 & 0.136 & $\begin{array}{l}26(55.3) \\
21(44.7)\end{array}$ & $<0.001$ & $<0.001$ \\
\hline \multicolumn{13}{|l|}{ Venous invasion } \\
\hline $\begin{array}{l}\text { No } \\
\text { YES }\end{array}$ & $\begin{array}{c}49(90.7) \\
5(9.3)\end{array}$ & $<0.001$ & $<0.001$ & $\begin{array}{c}92(100) \\
0(0)\end{array}$ & $\mathrm{NE}$ & $\mathrm{NE}$ & $\begin{array}{c}99(100) \\
0(0)\end{array}$ & $\mathrm{NE}$ & NE & $\begin{array}{r}42(89.4) \\
5(10.6)\end{array}$ & $<0.001$ & $<0.001$ \\
\hline \multicolumn{13}{|l|}{ BRAF mutation ${ }^{\text {b) }}$} \\
\hline $\begin{array}{l}\text { Negative } \\
\text { Positive }\end{array}$ & $\begin{array}{c}39(92.9) \\
3(7.1)\end{array}$ & 0.223 & 0.204 & $\begin{array}{c}62(67.4) \\
1(1.1)\end{array}$ & 0.483 & 0.672 & $\begin{array}{c}66(98.5) \\
1(1.5)\end{array}$ & 0.491 & 0.681 & $\begin{array}{c}35(92.1) \\
3(7.9)\end{array}$ & 0.277 & 0.278 \\
\hline
\end{tabular}

LNM, lymph node metastasis; CEA, carcinoembryonic antigen; CRT, chemoradiotherapy; RFS, recurrence free survival; CSS, cancer-specific survival, NE, not evaluated.

a) $p$-values were calculated using log-rank test. ${ }^{\text {b) } M i s s i n g ~ v a l u e ~ i s ~ i n c l u d e d . ~}$

Table 4. Multivariate analysis to determinate the independent prognostic factors in entire group with residual rectal cancer patients after preoperative CRT

\begin{tabular}{|c|c|c|c|c|c|c|c|}
\hline \multirow{2}{*}{ Variable } & \multirow{2}{*}{ Category } & \multicolumn{3}{|c|}{$\begin{array}{l}\text { Recurrence free survival } \\
\text { (multivariate analysis) }\end{array}$} & \multicolumn{3}{|c|}{$\begin{array}{l}\text { Cancer-specific survival } \\
\text { (multivariate analysis) }\end{array}$} \\
\hline & & $p$-value & $\mathrm{HR}$ & $95 \% \mathrm{Cl}$ & $p$-value & $\mathrm{HR}$ & $95 \% \mathrm{Cl}$ \\
\hline Pathologic TNM stage & III vs. I\&II & 0.105 & 1.744 & $0.890-3.418$ & 0.004 & 3.413 & $1.484-7.851$ \\
\hline Residual tumor size $(\mathrm{cm})$ & $\geq 6$ vs. $<6$ & 0.049 & 1.753 & $1.003-3.064$ & 0.020 & 2.371 & $1.143-4.919$ \\
\hline Venous invasion & Present vs. absent & $<0.001$ & 20.425 & $5.140-81.172$ & 0.086 & 2.767 & $0.865-8.854$ \\
\hline Lymphatic invasion & Present vs. absent & 0.889 & 1.050 & $0.528-2.089$ & 0.875 & 0.939 & $0.427-2.063$ \\
\hline Perineural invasion & Present vs. absent & 0.117 & 1.667 & $0.880-3.157$ & 0.009 & 2.634 & $1.271-5.458$ \\
\hline CRM & Positive vs. negative & $<0.001$ & 3.613 & $2.034-6.418$ & $<0.001$ & 4.133 & $2.027-8.426$ \\
\hline Tumor regression grade & $0 \& 1$ vs. $2 \& 3$ & 0.425 & 1.311 & $0.673-2.555$ & 0.368 & 1.404 & $0.670-2.941$ \\
\hline
\end{tabular}

CRT, chemoradiotherapy; HR, hazard ratio; $\mathrm{Cl}$, confidence interval; TNM, tumor, node, metastasis. vs., versus; CRM, circumferential resection margin. 
patients at a higher risk of disease progression, and may help clinicians implement more effective treatment strategies in patients presenting with residual rectal cancer after CRT.

\section{Acknowledgements}

This study was partly supported by a grant in-aid from Basic Science Research Program through the National Research Foundation of Korea (NRF) funded by the Ministry of Science, ICT and Future Planning (grant number: NRF2015R1C1A1A02037597).

\section{Conflicts of interest}

No potential conflicts of interest relevant to this article was reported.

\section{ORCID}

Sol-Min Kim, https://orcid.org/0000-0002-1299-153X Ghilsuk Yoon, https://orcid.org/0000-0002-9941-024X An Na Seo, https://orcid.org/0000-0001-6412-3067

\section{References}

1. Sannier A, Lefèvre JH, Panis Y, Cazals-Hatem D, Bedossa P, Guedj N. Pathological prognostic factors in locally advanced rectal carcinoma after neoadjuvant radiochemotherapy: analysis of 113 cases. Histopathology 2014;65:623-30.

2. Quah HM, Chou JF, Gonen M, Shia J, Schrag D, Saltz LB, et al. Pathologic stage is most prognostic of disease-free survival in locally advanced rectal cancer patients after preoperative chemoradiation. Cancer 2008;113:57-64.

3. Rödel C, Martus P, Papadoupolos T, Füzesi L, Klimpfinger M, Fietkau R, et al. Prognostic significance of tumor regression after preoperative chemoradiotherapy for rectal cancer. J Clin Oncol 2005;23:8688-96.

4. Medich D, McGinty J, Parda D, Karlovits S, Davis C, Caushaj P, et al. Preoperative chemoradiotherapy and radical surgery for locally advanced distal rectal adenocarcinoma: pathologic findings and clinical implications. Dis Colon Rectum 2001;44:1123-8.

5. Guillem JG, Chessin DB, Cohen AM, Shia J, Mazumdar M, Enker $\mathrm{W}$, et al. Long-term oncologic outcome following preoperative combined modality therapy and total mesorectal excision of locally advanced rectal cancer. Ann Surg 2005;241:829-36.

6. Duldulao MP, Lee W, Streja L, Chu P, Li W, Chen Z, et al. Distribution of residual cancer cells in the bowel wall after neoadjuvant chemoradiation in patients with rectal cancer. Dis Colon Rectum 2013;56:142-9.

7. Das P, Skibber JM, Rodriguez-Bigas MA, Feig BW, Chang GJ, Hoff PM, et al. Clinical and pathologic predictors of locoregional recurrence, distant metastasis, and overall survival in patients treated with chemoradiation and mesorectal excision for rectal cancer. Am J Clin Oncol 2006;29:219-24.

8. Bernstein TE, Endreseth BH, Romundstad P, Wibe A; Norwegian Colorectal Cancer Group. Circumferential resection margin as a prognostic factor in rectal cancer. $\mathrm{Br} \mathrm{J}$ Surg 2009;96:1348-57.

9. Rullier A, Laurent C, Capdepont M, Vendrely V, BioulacSage P, Rullier E. Impact of tumor response on survival after radiochemotherapy in locally advanced rectal carcinoma. Am J Surg Pathol 2010;34:562-8.

10. Gosens MJ, Klaassen RA, Tan-Go I, Rutten HJ, Martijn H, van den Brule AJ, et al. Circumferential margin involvement is the crucial prognostic factor after multimodality treatment in patients with locally advanced rectal carcinoma. Clin Cancer Res 2007;13:6617-23.

11. Gosens MJ, van Krieken JH, Marijnen CA, Meershoek-Klein Kranenbarg E, Putter H, Rutten HJ, et al. Improvement of staging by combining tumor and treatment parameters: the value for prognostication in rectal cancer. Clin Gastroenterol Hepatol 2007;5:997-1003.

12. Huebner M, Wolff BG, Smyrk TC, Aakre J, Larson DW. Partial pathologic response and nodal status as most significant prognostic factors for advanced rectal cancer treated with preoperative chemoradiotherapy. World J Surg 2012;36:67583.

13. Lee JH, Chie EK, Kim K, Jeong SY, Park KJ, Park JG, et al. The influence of the treatment response on the impact of resection margin status after preoperative chemoradiotherapy in locally advanced rectal cancer. BMC Cancer 2013;13:576.

14. Beddy D, Hyland JM, Winter DC, Lim C, White A, Moriarty M, et al. A simplified tumor regression grade correlates with survival in locally advanced rectal carcinoma treated with neoadjuvant chemoradiotherapy. Ann Surg Oncol 2008;15:3471-7.

15. Edge SB, Compton CC. The American Joint Committee on Cancer: the 7th edition of the AJCC cancer staging manual and the future of TNM. Ann Surg Oncol 2010;17:1471-4.

16. Kim HJ, Choi GS, Park JS, Park S, Kawai K, Watanabe T. Clinical significance of thrombocytosis before preoperative chemoradiotherapy in rectal cancer: predicting pathologic tumor response and oncologic outcome. Ann Surg Oncol 2015;22:513-9.

17. Park JS, Choi GS, Jun SH, Hasegawa S, Sakai Y. Laparoscopic 
versus open intersphincteric resection and coloanal anastomosis for low rectal cancer: intermediate-term oncologic outcomes. Ann Surg 2011;254:941-6.

18. Park JS, Huh JW, Park YA, Cho YB, Yun SH, Kim HC, et al. A circumferential resection margin of $1 \mathrm{~mm}$ is a negative prognostic factor in rectal cancer patients with and without neoadjuvant chemoradiotherapy. Dis Colon Rectum 2014;57:933-40.

19. Hwang MR, Park JW, Park S, Yoon H, Kim DY, Chang HJ, et al. Prognostic impact of circumferential resection margin in rectal cancer treated with preoperative chemoradiotherapy. Ann Surg Oncol 2014;21:1345-51.

20. Kwon MJ, Lee SE, Kang SY, Choi YL. Frequency of KRAS, BRAF, and PIK3CA mutations in advanced colorectal cancers: Comparison of peptide nucleic acid-mediated PCR clamping and direct sequencing in formalin-fixed, paraffin-embedded tissue. Pathol Res Pract 2011;207:762-8.

21. Nagtegaal ID, Quirke P. What is the role for the circumferential margin in the modern treatment of rectal cancer? J Clin Oncol 2008;26:303-12.

22. Quirke P, Durdey P, Dixon MF, Williams NS. Local recurrence of rectal adenocarcinoma due to inadequate surgical resection. Histopathological study of lateral tumour spread and surgical excision. Lancet 1986;2:996-9.

23. Birbeck KF, Macklin CP, Tiffin NJ, Parsons W, Dixon MF, Mapstone NP, et al. Rates of circumferential resection margin involvement vary between surgeons and predict outcomes in rectal cancer surgery. Ann Surg 2002;235:449-57.
24. Wibe A, Rendedal PR, Svensson E, Norstein J, Eide TJ, Myrvold $\mathrm{HE}$, et al. Prognostic significance of the circumferential resection margin following total mesorectal excision for rectal cancer. Br J Surg 2002;89:327-34.

25. Baik SH, Kim NK, Lee YC, Kim H, Lee KY, Sohn SK, et al. Prognostic significance of circumferential resection margin following total mesorectal excision and adjuvant chemoradiotherapy in patients with rectal cancer. Ann Surg Oncol 2007; 14:462-9.

26. de Haas-Kock DF, Baeten CG, Jager JJ, LangendijkJA, Schouten LJ, Volovics A, et al. Prognostic significance of radial margins of clearance in rectal cancer. Br J Surg 1996;83:781-5.

27. Bouzourene H, Bosman FT, Matter M, Coucke P. Predictive factors in locally advanced rectal cancer treated with preoperative hyperfractionated and accelerated radiotherapy. Hum Pathol 2003;34:541-8.

28. Luna-Pérez P, Bustos-Cholico E, Alvarado I, Maffuz A, Rodríguez-Ramírez S, Gutiérrez de la Barrera M, et al. Prognostic significance of circumferential margin involvement in rectal adenocarcinoma treated with preoperative chemoradiotherapy and low anterior resection. J Surg Oncol 2005;90:20-5.

29. Rullier A, Gourgou-Bourgade S, Jarlier M, Bibeau F, ChassagneClément C, Hennequin C, et al. Predictive factors of positive circumferential resection margin after radiochemotherapy for rectal cancer: the French randomised trial ACCORD12/0405 PRODIGE 2. Eur J Cancer 2013;49:82-9. 